\title{
Relações Interpessoais, Equipe de Trabalho e seus Reflexos na Atenção Básica
}

\author{
Interpersonal Relations, Teamwork, and the \\ Impact on Primary Care
}

\author{
Alexandra da Rosa Martins \\ Denise Bermudez Pereira \\ Maria Laura Silveira Nogueira Nogueiral \\ Celeste dos Santos PereiralI \\ Greice Schrader Schrader \\ Maira Buss Thoferhn ${ }^{I I}$
}

\author{
PALAVRAS-CHAVE: \\ - Relações Interpessoais; \\ - Equipe Interdisciplinar \\ de Saúde; \\ - Atenção Básica.
}

Recebido em: 20/09/2010

Aprovado em: 19/11/2010
REVISTA BRASILEIRA DE EDUCAÇÃO MÉDICA $6 \frac{\text { Lupl. 2): 6-12; 2012 }}{36(1, \text { }}$

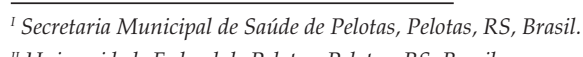

II Universidade Federal de Pelotas, Pelotas, RS, Brasil..

\begin{abstract}
A formação e afirmação de vínculos profissionais têm por finalidade o desenvolvimento de relações interpessoais em equipes de trabalho, compreendendo a sua realidade para promover o crescimento de cada um dos profissionais individualmente e do grupo de trabalho como um todo, facilitando a resolução dos conflitos. O presente trabalho teve por objetivo resgatar as relações de trabalho em uma equipe multiprofissional para a formação de vínculos profissionais saudáveis. Foi realizada uma pesquisa convergente-assistencial, adotando-se uma abordagem construtivista de caráter qualitativo. O estudo foi feito em uma USF localizada na periferia da cidade de Pelotas - RS. Os sujeitos desse estudo foram nove profissionais, entre eles: dois médicos, três enfermeiros, três auxiliares de enfermagem e uma assistente social, que contaram ainda com dois acadêmicos da graduação em Enfermagem da UFPel. Portanto, foi possível vivenciar a experiência da Teoria dos Vínculos Profissionais, que proporcionou ao grupo momentos de reflexão a respeito da dinâmica das relações na equipe multiprofissional, permitindo com se alcançasse o objetivo desejado.
\end{abstract}

The creation and affirmation of professional links aims to develop interpersonal relations in work teams, understanding their reality in order to foster the professionals' individual growth and that of the team as a whole, thereby facilitating conflict resolution. The current study aimed to reclaim the work relations in a multidisciplinary team in order to form healthy professional links. A convergent care study was performed using a constructivist qualitative methodology. The study was conducted in a Family Health Unit located on the periphery of the city of Pelotas, Rio Grande do Sul State, Brazil. The study subjects were nine health professionals: two physicians, three nurses, three nurse technicians, and one social worker, plus two undergraduate nursing students. It was thus possible to witness an experience with the Theory of Professional Links, providing the group with moments of reflection on the dynamics of multidisciplinary team relations and allowing the study to achieve its objective. 


\section{INTRODUÇÃO}

Atenção Básica à Saúde (ABS) caracteriza-se por um conjunto de ações visando à promoção e proteção da saúde, prevenção de agravos, tratamento e reabilitação, e manutenção da saúde do indivíduo e da coletividade ${ }^{1}$. É entendida como a porta de entrada no Sistema de Saúde e tem como princípios norteadores a garantia do acesso universal e equânime da população, assim como o atendimento integral e descentralizado da saúde ${ }^{2}$.

O desenvolvimento da ABS requer o trabalho de uma equipe multiprofissional, com habilidades e conhecimento técnico, capaz de identificar e resolver os problemas de saúde de maior frequência e relevância no território sob sua responsabilidade ${ }^{1}$.

Atualmente, a Estratégia Saúde da Família (ESF) é o principal instrumento para a reorganização da Atenção Básica em nosso país. Inicialmente denominada Programa Saúde da Família (PSF), foi lançada pelo governo federal em 1994, tendo como objetivo a reorientação do modelo de atenção, com ênfase na promoção, prevenção e recuperação da saúde das famílias, buscando contribuir para a consolidação do Sistema Único de Saúde (SUS).

A Estratégia possibilita a integração e promove a organização das atividades em um território definido, com o intuito de propiciar o enfrentamento e a resolução dos problemas identificados. Representa uma mudança em relação ao modelo tradicional, com a integração das ações e serviços de saúde. Além da facilidade de ter uma população adscrita e territorializada, tendo a oportunidade, portanto, de conhecer sua comunidade na totalidade, tem a potencialidade de contribuir para que as ações de saúde se apresentem de forma mais efetiva ${ }^{3}$.

A ESF, estruturada na lógica da Atenção Básica à Saúde, gera novas práticas e necessita desenvolver processos de trabalho que estabeleçam uma nova relação entre a comunidade e os profissionais de saúde. O trabalho em equipe que se desenvolve na ESF demanda ainda a integração de ações dos profissionais da equipe de saúde por meio de um processo de interação e de articulação das intervenções técnicas ${ }^{4}$

No final de 2002, foi implantado o Programa Saúde da Família (PSF) em uma unidade básica de saúde localizada na periferia da cidade de Pelotas - RS. Essa Unidade de Saúde da Família (USF) é composta por três equipes, cada uma constituída por um médico, um enfermeiro e um auxiliar de enfermagem, além de agentes comunitários de saúde. As equipes contam ainda com o apoio de dois dentistas, um assistente social, um nutricionista, quatro recepcionistas e três higienizadores, representando a equipe de apoio.
No que tange às relações entre os profissionais do serviço, pode-se afirmar que, nos primeiros dois anos de convívio, os laços afetivos entre eles eram estreitos, e os problemas de relacionamento eram facilmente resolvidos, pois existia cooperação, solidariedade, amizade. As decisões eram tomadas em conjunto, sempre respeitando a individualidade e a singularidade dos colegas, ou seja, havia vínculos profissionais saudáveis. Ocorria um encontro mensal, fora do horário de trabalho, para descontração e integração das equipes e seus familiares.

Com o passar do tempo, houve um desgaste das relações grupais, e a equipe acabou perdendo sua identidade, a característica própria que havia conquistado. O grupo foi se desmotivando por problemas relacionados à gestão, como: questões trabalhistas, incluindo remuneração inadequada, falta de credibilidade, apoio e ferramentas de trabalho. $\mathrm{O}$ fato de haver a substituição de alguns membros da equipe, que ingressaram com dificuldades de trabalhar coletivamente, assim como o surgimento de problemas de comunicação, gerou incompreensões, mágoas e ressentimentos, aumentando o distanciamento entre os trabalhadores da unidade.

Cabe ressaltar que em um grupo de trabalho ocorrem trocas internas - da pessoa consigo mesma -, e trocas externas - dela com o grupo e o ambiente. Essas trocas são constantes e geram um comportamento que é próprio de cada grupo. As modificações abruptas nessas trocas, tanto internas quanto externas, ocasionam um desequilíbrio grupal. Dessa forma, a alteração dos sujeitos-trabalhadores, seja por rodízios institucionais ou por iniciativa de alguns participantes, acarreta o inevitável reajuste grupal que determina a necessidade de propor novos compromissos e redistribuição de ações previamente determinadas ${ }^{5}$.

Para Thofehrn e Leopardi, a formação e afirmação de vínculos profissionais têm por objetivo o desenvolvimento de relações interpessoais em cada equipe de enfermagem, compreendendo a realidade a ser trabalhada para promover o crescimento de cada um e de todo o grupo de trabalho, facilitando a resolução dos conflitos, ao mesmo tempo em que propicia o desenvolvimento pessoal. Assim, entende-se que vínculo profissional é uma relação humana entre trabalhadores, reunidos para um trabalho em conjunto, visando a atingir as mesmas finalidades e objetivos.

Para que haja a formação de vínculos, o grupo de trabalho deve ser maleável, receptivo e adaptável às contínuas modificações que ocorrem nos serviços de saúde. Nesse processo de formação de vínculos, deve-se lembrar que as pessoas são diferentes, com posições individuais e coletivas que devem ser respeitadas, podendo ainda acontecer entre a equipe conflitos e adversidades, que devem ser solucionados mediante partici- 
pação e comprometimento de todo o grupo ${ }^{5}$. Acredita-se que o comprometimento com o trabalho propicia uma abertura e um contato entre as pessoas, estabelecendo uma relação de confiança.

A Teoria dos Vínculos Profissionais (TVP) ${ }^{6}$, utilizada para embasar este estudo, tem o propósito de formar vínculos entre os membros da equipe de enfermagem. No entanto, ampliou-se esta proposta para além desse grupo específico, aplicando-a a toda a equipe multiprofissional.

Sendo o local do estudo uma USF vinculada ao ensino, que acolhe alunos dos cursos de Enfermagem, Nutrição e Medicina da Universidade Federal de Pelotas (UFPel), procurou-se sempre envolver os acadêmicos em todos os aspectos inerentes ao processo de trabalho da equipe, buscando contribuir para a formação de profissionais generalistas, críticos, reflexivos e competentes em suas práticas e responsáveis ética e socialmente, como prevê o Projeto Institucional de Reorientação da Formação Profissional em Saúde (Pró-Saúde). Entende-se que é necessário investir na qualificação do grupo de trabalho, envolvendo alunos, professores e profissionais de saúde em temas com metodologias ativas, trabalho em pequenos grupos e avaliação somativa ${ }^{7}$.

É importante ressaltar que o presente relato de experiência é fruto de um trabalho desenvolvido em 2008 com alguns profissionais da equipe de saúde, contando com a participação de alunos da graduação em Enfermagem. Atualmente, estão sendo desenvolvidos na USF os projetos Pró-Saúde e PET-Saúde (Programa de Educação para o Trabalho em Saúde) ${ }^{11}$. A referida USF, por estar atrelada ao ensino, recebe acadêmicos de diversos campos da saúde, como Enfermagem, Nutrição e Medicina, como citado anteriormente. Somam-se, hoje, 35 alunos do PET-Saúde e 28 alunos dos estágios curriculares da Enfermagem, distribuídos durante a semana nos dois turnos de atendimento.

Em virtude do grande volume de acadêmicos que hoje se adicionam à equipe, sentiu-se a necessidade de retomar o trabalho ora iniciado, buscando melhorar as relações de trabalho e, consequentemente, oferecer maior qualidade no atendimento aos usuários.

Dessa forma, o presente estudo teve como objetivo resgatar as relações de trabalho em uma equipe multiprofissional, para a formação de vínculos profissionais saudáveis.

\section{METODOLOGIA}

Foi realizada uma pesquisa convergente-assistencial, adotando-se uma abordagem construtivista de caráter qualitativo. Nesse tipo de estudo, propõe-se a articulação da prática assistencial com o conhecimento teórico, assim como a refle- xão dessa prática a partir de fenômenos vivenciados no seu contexto ${ }^{8}$.

Na pesquisa convergente-assistencial, o enfermeiro sustenta uma estreita relação com a condição social, buscando soluções para problemas, propondo mudanças e inovações, ou seja, compromete-se com o avanço do contexto social estudado. Nessa pesquisa, o profissional e o pesquisador unem o pensar e o saber, que conduz ao "saber fazer" no seu cotidiano, constituindo-se um método apropriado para investigar a prática assistencial da enfermagem ${ }^{8}$.

Configura-se por ser um método alternativo que prima pela flexibilidade, expondo a sensibilidade da relação entre investigador, pesquisador e referencial teórico. É pautado em uma abordagem humanística, com estímulo à criatividade e respeito à individualidade.

Esta pesquisa teve uma abordagem qualitativa, pois buscou compreender o problema a partir da perspectiva dos sujeitos que o vivenciam, partindo-se de sua vida diária, suas emoções, seus sentimentos e desejos, atentando-se ao contexto social no qual ocorre o evento9.

O estudo foi realizado em uma USF localizada na periferia da cidade de Pelotas - RS, onde a ESF está implantada há oito anos. Os sujeitos do estudo foram nove profissionais, entre eles dois médicos, três enfermeiros, três auxiliares de enfermagem e uma assistente social, além de dois acadêmicos da graduação em Enfermagem da UFPel.

O estudo seguiu os procedimentos éticos exigidos pela resolução no 196/96 do Conselho Nacional de Saúde do Ministério da Saúde. Foi aprovado pelo Comitê de Ética da UFPel e pela coordenação do Departamento de Saúde Pública da Secretaria Municipal de Saúde e Bem-Estar. Todos os participantes concordaram em fazer parte do estudo, assinando o Termo de Consentimento Livre e Esclarecido permitindo que, após a análise dos dados, os resultados fossem divulgados nos meios científicos.

Para a implementação desta prática foi utilizada a técnica de grupo focal, na qual os dados são obtidos diretamente das falas de um grupo, no momento em que ele descreve suas experiências e percepções em torno de um tema de interesse coletivo9.

Foram realizados cinco encontros, com o intuito de implantar a TVP no grupo de trabalho. Os encontros aconteceram durante os meses de dezembro de 2007, janeiro e fevereiro de 2008 na própria USF e respeitaram as seguintes etapas:

a) Apresentação e aceitação da proposta.

b) Preparação do grupo para trabalhar os vínculos profissionais.

c) Resgate dos laços afetivos.

d) Construção do processo de trabalho em equipe.

e) Fechamento do grupo. 


\section{ANÁLISE DOS DADOS}

\section{Encontro - Apresentando a proposta}

O primeiro encontro foi iniciado com a apresentação à equipe multiprofissional da proposta de implantação da Teoria dos Vínculos Profissionais (TVP) no grupo de trabalho. Em seguida, utilizou-se uma técnica de dinâmica de grupo na qual os participantes receberam balões contendo mensagens. Após o momento de brincadeira e descontração, cada um estourou seu balão e leu a mensagem para os outros. Esta atividade teve como objetivo promover uma breve reflexão acerca das relações humanas no trabalho.

Nos instantes seguintes, observou-se o silêncio por parte de alguns integrantes e também algumas fisionomias sérias. Alguns participantes apenas fizeram a leitura das frases sem tecer comentários, enquanto outros não só discutiram como relacionaram algumas ideias, refletindo sobre a necessidade do autoconhecimento na construção de relações de trabalho saudáveis. Nesse contexto, percebeu-se que, para que o grupo construa relações de trabalho saudáveis, além do fato de ser indispensável conhecer-se, é imprescindível que identifique suas características, refletindo sobre a necessidade de modificar suas relações ${ }^{5}$.

Nesse primeiro encontro, como a intenção foi sensibilizar o grupo para trabalhar o tema proposto, pode-se afirmar que o resultado foi positivo. A equipe acolheu a proposta de implantação da Teoria dos Vínculos Profissionais, valorizando a importância de abordar as relações humanas no ambiente de trabalho.

Discutiu-se com a equipe as possibilidades e os horários para os próximos encontros. Foi distribuída uma ficha de avaliação sugerindo-se ressaltar pontos positivos, negativos e sugestões. A partir da análise das fichas, pode-se citar como pontos positivos o estímulo à equipe para refletir o grande grupo; a discussão e o pensar das relações; e o momento para a integração, reflexão e mudanças. Como ponto negativo, um integrante destacou sua dificuldade para discutir as relações de trabalho; desse modo, foi sugerida a utilização de dinâmicas antes das discussões.

Ao término desse encontro, entendeu-se que o grupo aprovou, como afirmam Thoferhn e Leopardi5, a proposta como um modelo para ser utilizado na equipe, percebendo a necessidade da organização do trabalho, bem como a importância de se oferecer às pessoas um cuidado terapêutico de qualidade, em busca de relações mais afetuosas, humanas e sensíveis entre os profissionais.

\section{Encontro - Fortalecendo vínculos}

O segundo encontro foi iniciado com um trabalho de relaxamento e sensibilização, com o objetivo de fortalecer os vínculos profissionais e estimular o planejamento de atividades para atingir objetivos e metas. Discutiu-se também a expressão vínculo profissional, entendida como uma relação de trabalho na qual o grupo reflete comprometimento, cumplicidade, respeito, afeto e flexibilidade, enquanto desenvolve suas ações de saúde, objetivando um cuidado terapêutico de qualidade. O grupo foi dividido em duas equipes. Uma equipe recebeu um balão, tendo como tarefa protegê-lo da outra, que deveria estourá-lo.

A equipe que deveria estourar o balão organizou-se rapidamente, definindo o ataque, enquanto a outra não parou para se organizar. Somente um integrante desta equipe tirou o relógio para evitar o estouro.

Após a dinâmica, foi questionado ao grande grupo o significado da atividade. Cada componente interpretou de forma diferente, apenas um identificou a necessidade de planejar e organizar as ações do grupo. Houve indignação do grupo protetor, que protestou contra o fato de não terem tido tempo para se organizar.

Para Thoferhn e Leopardi ${ }^{5}$, o planejamento de ações deve existir e ser participativo, ou seja, todos os membros da equipe precisam envolver-se, desde a elaboração, aprovação, execução e avaliação, objetivando o alcance da tarefa profissional no processo de trabalho. Para as autoras, o planejamento é importante para formação e afirmação dos vínculos profissionais, favorecendo o comprometimento e envolvimento de toda a equipe.

Percebeu-se que nesse encontro houve interação, descontração e participação efetiva de todos, resgatando alguns membros que haviam ficado distantes no primeiro encontro. Desse modo, entende-se que essa etapa correspondeu à fase de inclusão do desenvolvimento grupal da Teoria dos Vínculos, na qual ocorre a estruturação do grupo.

Outras duas zonas de necessidades interpessoais devem estar presentes em um grupo: a fase de controle, na qual as pessoas dão sugestões e participam da tomada de decisões; e a fase de abertura, na qual manifestam laços emocionais, apoio, afeto, momentos de satisfação e insatisfação. A repetição dessas fases pode ocorrer independentemente do tempo de relação e duração do grupo. Deve-se ficar atento para que as relações e o grupo não se desfaçam, para que o afeto não deixe de ser expresso, as relações não fiquem frouxas e as pessoas não sintam necessidade de se excluírem6 .

\section{3 - Encontro - Reforçando laços afetivos}

Nesse encontro, foi sugerido ao grupo iniciar com a dinâmica dos bonecos, com o objetivo de reforçar os laços afetivos entre os membros da equipe. Foi explicado ao grupo o desenvolvimento da dinâmica, na qual cada participante 
recebe dois bonecos, com contornos de rosto e corpo, possibilitando a escrita. No corpo do primeiro boneco, deve ser escrita uma qualidade pessoal e, na cabeça, um aspecto negativo. Em seguida, todos devem recortar a cabeça e colocá-la em um saco plástico, que deverá passar por todos os participantes para que retirem uma cabeça. Todos devem pegar o segundo boneco e expressar uma reflexão acerca da dificuldade do colega. Na apresentação, cada um relata sua qualidade, apresentando no segundo boneco sua sugestão ao colega.

No primeiro momento, alguns não entenderam o que fariam com o boneco. Novamente, explicou-se o que deveriam fazer em cada etapa.

Todos participaram do desenvolvimento da atividade, refletindo sobre a dificuldade de seu colega de trabalho e sugerindo técnicas, mudanças de atitudes, de comportamento, dicas simples como auxílio para a superação das dificuldades apresentadas. Durante essa atividade, observou-se o envolvimento das pessoas, a preocupação com o colega, um sentimento de respeito mútuo, indo ao encontro da Teoria dos Vínculos Profissionais, a qual faz referência à fase de afeição, revelando as expressões de interação emocional, com manifestação de apoio e afeto.

Um ponto forte desse encontro foi a participação efetiva de um membro da equipe que, anteriormente, mantinha-se distante.

\section{4ำ Encontro - Construindo o processo de trabalho}

$\mathrm{O}$ quarto encontro da equipe multiprofissional teve como objetivo a construção do processo de trabalho do grupo. No primeiro momento, apresentou-se de forma resumida o conjunto de elementos que constituem o processo de trabalho da enfermagem. A seguir, foi proposto o desafio de elaborar, em cartazes, o que o grupo entendia por tarefa profissional, objeto de trabalho, força de trabalho, finalidade do trabalho e instrumento de trabalho da equipe multiprofissional.

O grupo realizou a tarefa refletindo sobre o cuidado dispensando aos usuários, buscando compreender as relações entre clientes, trabalhadores e instituição de saúde. A construção se deu de forma participativa, havendo a discussão de cada item em que todos verbalizaram suas opiniões.

Com o mesmo objetivo agregador deste trabalho, e considerando o descrito anteriormente, salienta-se que o Pró-Saúde tem como proposta a superação de antigos métodos de ensino, investindo em experiências inovadoras, como a incorporação da problematização de situações e o trabalho em pequenos grupos. A seguir, descreve-se o resultado do processo de trabalho elaborado pela equipe.
O processo de trabalho da equipe multiprofissional é entendido como um conjunto de ações integradas desenvolvidas pelos membros da equipe de saúde, objetivando atender às necessidades dos indivíduos e famílias da comunidade. Envolve os saberes dos vários profissionais que compõem a equipe (enfermeiros, médicos, auxiliares e técnicos de enfermagem, assistente social, nutricionista, agente comunitário de saúde, psicólogos, alunos, professores, entre outros).

A tarefa profissional da equipe de saúde foi definida como o cuidado integral prestado aos clientes (indivíduos, famílias e comunidade), buscando atender alguma necessidade de saúde, levando a uma melhor qualidade de vida.

O objeto de trabalho foi descrito como sendo os usuários do serviço de saúde, bem como a comunidade na qual estão inseridos. São os indivíduos que necessitam de ações terapêuticas, tanto preventivas quanto curativas.

Como instrumentos ou ferramentas de trabalho a equipe citou os recursos materiais, incluindo todos os equipamentos disponíveis; as estratégias utilizadas para promover a educação em saúde, desde os atendimentos individuais, grupos específicos até os eventos educativos nas escolas e comunidade; e o gerenciamento da unidade. Todas as condutas que permitem desenvolver as ações de saúde foram consideradas ferramentas de trabalho.

A força de trabalho consiste na soma das competências de todos os trabalhadores que compõem a equipe multiprofissional, com a parceria da comunidade acadêmica.

Como finalidade do trabalho, a equipe entende a promoção de uma melhor qualidade de vida e saúde por meio da prestação de um cuidado adequado aos usuários.

Considerando-se o que foi construído pelos participantes, entende-se que o grupo percebe-se envolvido em um processo coletivo de trabalho, em uma perspectiva de complementaridade das ações, na qual todos os membros da equipe têm sua importância e valor reconhecidos. De acordo com Ribeiro et al. ${ }^{10}$, desenvolver um trabalho em equipe representa a conexão entre os diversos processos de trabalho implicados, havendo a necessidade de reconhecer e valorizar o trabalho do outro, considerando sua participação na produção do cuidado. Significa ainda elaborar consensos em relação às metas que se deseja atingir, valendo-se da interação entre os atores envolvidos em busca de compreensão e respeito mútuo dos saberes e da autonomia técnica.

Após a elaboração e discussão do processo de trabalho, a equipe debateu sobre a forma pela qual se estabeleceu a relação de trabalho enquanto grupo e como foram construídos os vínculos. Foi consenso entre os membros da equipe que a satisfação pessoal está intimamente relacionada com a satisfação 
profissional e a harmonia entre os colegas de trabalho. Para Thoferhn e Leopardi ${ }^{5}$, não é possível conceber a pessoa em sua plenitude separada de suas ações profissionais, já que é muito difícil instituir um mundo independente da conjuntura de trabalho. O que é possível é a realização do trabalho objetivando obter a realização pessoal.

\section{$5^{\circ}$ Encontro - Fechando o grupo de trabalho}

No último encontro, foi sugerido ao grupo descrever em cartazes, de forma resumida, todos os assuntos desenvolvidos anteriormente, de modo a retomar os aspectos já trabalhados e discutir outros que o grupo entendesse como relevantes, objetivando a consolidação dos vínculos profissionais saudáveis.

A equipe multiprofissional foi dividida em dois pequenos grupos, que receberam cartolinas, revistas, pincéis, cola e tesouras. Os grupos trabalharam por alguns minutos e, depois, apresentaram aos colegas suas construções.

O primeiro grupo reforçou, em forma de figuras e frases, que a equipe de trabalho deve manter-se unida e que, considerando a singularidade de cada um, deve ter espírito de aventura, superar as limitações e enfrentar obstáculos, buscando os mesmos objetivos, crescendo juntos e fortalecendo seus vínculos de trabalho, evitando situações que impeçam o crescimento coletivo.

O segundo grupo também se expressou com figuras e frases, dando ênfase ao processo de trabalho articulado ao vínculo profissional, resultando em uma melhor qualidade de vida, tanto para os trabalhadores como para a comunidade assistida. Salientaram ainda que todos os profissionais devem contribuir para a manutenção e continuidade do espírito de equipe, sendo necessária uma reflexão individual de forma a contribuir com o processo coletivo de fortalecimento do grupo.

Ao final da atividade, foi advertido ao equipe que aquele seria o último encontro, mas que a proposta dos vínculos profissionais seria mantida durante as reuniões semanais de trabalho, considerando-se a boa aceitação dos profissionais.

\section{CONSIDERAÇÕES FINAIS}

A partir do desenvolvendo a proposta da Teoria dos Vínculos Profissionais, de Thoferhn e Leopardi ${ }^{5}$, junto à equipe, constatou-se ser imprescindível nas relações profissionais conhecer-se e conhecer o outro na sua individualidade, a fim de estar disponível para relacionar-se com as demais pessoas.

Vivenciar a experiência da teoria oportunizou ao grupo momentos de reflexão a respeito da dinâmica das relações na equipe multiprofissional, promovendo o resgate das relações de trabalho e a formação de vínculos profissionais saudáveis.

Partindo do pressuposto de que as relações interprofissionais tornaram-se consideravelmente mais respeitosas e colaborativas a partir da aplicação da Teoria dos Vínculos Profissionais na equipe, recomeçou-se a aplicá-la no momento em que o grupo foi igualmente se ampliando. Como a concentração de pessoas trabalhando juntas é maior pela chegada de acadêmicos de diversas áreas e com pensamentos distintos, também é mais frequente o aparecimento de divergências. Como a equipe sentiu-se unida e fortalecida, o desenrolar do processo de trabalho está sendo compartilhado com bom relacionamento entre todos e com melhores resultados, ou seja, mais qualidade no ambiente de trabalho acarretando um cuidado mais eficaz ao usuário.

Tornou-se necessária e indispensável a presença dos acadêmicos, preceptores e tutores nas reuniões semanais para avaliação e organização do serviço, considerando-se as sugestões e críticas de todos, pois entende-se que todos fazem parte do processo de trabalho e são igualmente importantes.

É interessante considerar que esse exercício deve ser dinâmico, atualizando-se e fortalecendo os vínculos continuamente. Problemas surgem diariamente e as formas como são superados são reflexos de quão unida e amadurecida encontra-se a equipe que os enfrenta. Nesse contexto, a comunidade acadêmica, agora inserida nesse grupo, vai se comportar de acordo com o exemplo que ela vê. Se encontrar um grupo coeso e unido, fica óbvio que deve seguir os "ensinamentos" dele. Então, esse grupo estará ajudando a formar profissionais aptos a trabalhar verdadeiramente em equipe, sem esquecer que a nova proposta de formação deseja e estimula a formação de indivíduos críticos e pró-ativos, o que significa que se espera que os alunos sejam capazes de compreender o universo em que estão inseridos e possam fazer sugestões para qualificar esses espaços.

A teoria é uma proposta para a formação e afirmação de vínculos profissionais junto à equipe de enfermagem, mas se ousou adaptá-la à equipe multiprofissional, obtendo sucesso, já que proporcionou o crescimento individual e coletivo desta, gerando integração, motivação, comprometimento e consciência da importância dos vínculos profissionais.

Espera-se que este estudo possa contribuir significativamente para outras equipes multiprofissionais que estejam dispostas a trabalhar com esse tema complexo que envolve as relações interpessoais, visto que muitas instituições públicas de saúde não abordam a dinâmica das relações de trabalho de forma reflexiva e participativa. 


\section{REFERÊNCIAS}

1. Ministério da Saúde. Portaria no 648, de 28 de março de 2006. M. D. Saúde. Brasília: Ministério da Saúde, 2006.

2. Facchini LA. Desempenho do PSF no Sul e Nordeste do Brasil: avaliação institucional e epidemiológica da Atenção Básica à Saúde. Ciênc. saúde coletiva 2006; 11(3): 669-681.

3. Ministério da Saúde. Saúde da Família. Uma estratégia para reorientação do modelo assistencial. Brasília: MS; 1997.

4. Ermel RC, Fracolli LA. O trabalho das enfermeiras no Programa de Saúde da Família em Marília/SP. Rev Esc Enferm USP 2006; 40(4):533-539.

5. Thofehrn MB, Leopardi MT. Teoria dos Vínculos Profissionais: um novo modo de gestão em enfermagem. Rev Texto \& Contexto enferm 2006; 15(3): 409-17.

6. Thofehrn MB. Vínculos Profissionais: uma proposta para o trabalho em equipe na enfermagem. Florianópolis; 2005. [Tese] - Programa de Pós-Graduação em Enfermagem, UFSC.

7. Brasil. Ministério da Saúde. Ministério da Educação. Programa Nacional de Reorientação da Formação Profissional em Saúde - Pró-Saúde: objetivos, implementação e desenvolvimento potencial. Brasília: Ministério da Saúde, 2007 [Série C. Projetos, Programas e Relatórios]

8. Trentini M, Paim L. Pesquisa em Enfermagem: uma modalidade convergente-assistencial. Florianópolis: Ed UFSC; 1999.

9. Leopardi MT. Metodologia de pesquisa em saúde. Santa Maria: Pallotti; 2001.

10. Ribeiro MR, Pires D, Blank VL. A teorização sobre o processo de trabalho em saúde como instrumental para análi- se do trabalho no Programa Saúde da Família. Cad Saúde Pública 2004; 20(2): 438-446.

11. Programa Nacional de Reorientação da Formação Profissional em Saúde - Pró-Saúde: apresentação. Disponível em: <http: / / prosaude.org/not/prosaude-maio2009/proSaude.pdf $>$ Acessado em: 17/09/2010.

\section{CONTRIBUIÇÃO DOS AUTORES}

Alexandra da Rosa Martins atuou ativamente em todas as fases de desenvolvimento do artigo. Denise Bermudez Pereira participou da construção e implementação do projeto "Educancdo para a Saúde", também atuou ativamente em todas as fases de desenvolvimento e organização do artigo. Maria Laura Silveira Nogueira contribuiu no desenvolvimento da metodologia, resultados e conclusão. Celeste dos Santos Pereira participou de todas as etapas do trabalho e revisão do mesmo. Greice Schrader auxiliou na revisão de literatura e confecção do artigo. Maira Buss Thoferhn contribuiu na construção do artigo.

\section{CONFLITO DE INTERESSES}

Declarou não haver.

\section{ENDEREÇO PARA CORRESPONDÊNCIA}

Alexandra da Rosa Martins

Rua Dr.Osvaldo Branco de Araújo, 111

Areal - Pelotas

CEP. 96085-355 RS

E-mail: alexandrarosamartins@hotmail.com 